“C 2006 IEEE. Personal use of this material is permitted. Permission from IEEE must be obtained for all other uses, in any current or future media, including reprinting/republishing this material for advertising or promotional purposes, creating new collective works, for resale or redistribution to servers or lists, or reuse of any copyrighted component of this work in other works." 


\title{
Control of Plasmon Resonance in Coatings of Gold Nanorods
}

\author{
Michael Cortie and Xiaoda Xu \\ Institute for Nanoscale Technology \\ University of Technology Sydney, Broadway NSW 2007, Australia \\ Email : michael.cortie@uts.edu.au
}

\begin{abstract}
Gold nanorods manifest a tunable plasmon resonance with light in the visible to near-infrared regions of the spectrum, and have been proposed for use in spectrally selective coatings on glass. However, details of shape and packing density have a significant effect on the optical properties of these nanoparticle coatings. Here we show how these effects can be controlled and exploited to produce a flexible spectral response.
\end{abstract}

Keywords- gold, nanorod, plasmon, optical extinction, spectral selectivity

\section{INTRODUCTION}

The widespread interest in the optical properties of precious metal nanoparticles of unusual shape, such as nanorods, nanotriangles, nanoshells and nanocaps, has inspired us to consider whether gold nanoparticles of these shapes might have applications in solar glazing or colour-change coatings. Unlike solid gold nanospheres (which manifest only a transverse plasmon resonance at $\sim 525 \mathrm{~nm}$ ), these new shapes exhibit additional localised surface plasmon resonances that depend sensitively on their geometry. Nanorods, for example, have the usual 'transverse' surface plasmon peak and a second, 'longitudinal', peak at longer wavelengths. The position of this second peak can be tuned from the mid-visible $(550 \mathrm{~nm})$ into the infrared $(1200 \mathrm{~nm})$ area by increase of the aspect ratio of the rod [1], with further adjustment possible by controlling the details of shape and symmetry [2]. In the case of rods it is the longitudinal peak that provides the possibility of improved spectral selectivity between the visible and IR regions, or of a switchable colour-display system, e.g. [3].

The process to make rods is now comparatively well known [1,4], although nobody, as far as we are aware, has scaled production up yet to commercially meaningful quantities. In addition, while there have been several reports of coatings produced with rods [5-8], there is not yet any dominant protocol to achieve this. A factor that has only recently received attention is that the individual plasmon resonances of gold nanorods in colloidal suspension are modified in high density coatings of these particles. The reason is that dipole-dipole interactions occur between metallic nanoparticles when they are sufficiently close together (see for example Peceros et al.[9] and references therein). In general, these interactions lead to a red-shifting of the extinction spectra, but this is not invariably the case. Furthermore, we will show that these factors can have a significant effect on the optical properties of coatings comprised of rods and must necessarily be taken in account.

\section{EXPERIMENTAL}

The optical properties of gold nanorods were numerically simulated using the DDSCAT code of Draine and Flatau [10, 11]. This is based on the discrete-dipole approximation method (DDA). The advantage of DDA is that it is an approximation method which converts the continuum target into a finite array of polarizable points, which acquire dipole moments in response to the local electric fields. Therefore, it can in principle be applied to any shape of particle. A disadvantage of DDA is that it is very computation intensive, and this makes some problems tedious to solve, even with the support of cluster or supercomputing computer hardware. For the present work a front-end program to generate the various configurations of rods and their associated input files was developed in VisualBASIC. The coupling between rods is simulated by generating a comparatively large input file that contains two nanorods, oriented and separated as required, and set into a larger cube shape, the remaining cells of which are notionally occupied by the medium. For convenience the optical properties are given in terms of an extinction efficiency, $Q_{\text {ext }}$, which is the wavelength-dependent optical cross-section normalized by the effective cross-sectional area of the particle.

The effect of $\mathrm{Ag}^{+}$on the aspect ratio of rods was investigated by producing rods using the seed-mediated growth method [12]. A 'seed' solution of spherical gold nuclei was first prepared by mixing $0.10 \mathrm{ml}$ of $0.02 \mathrm{M} \mathrm{KBH}_{4}$ with $10.0 \mathrm{ml}$ of an aqueous solution containing $0.5 \mathrm{mM} \mathrm{HAuCl}_{4}$ and $0.2 \mathrm{M}$ CTAB. ( $\mathrm{NaBH}_{4}$ may also be used and works as well.) After vigorous stirring for 30 minutes $12 \mu \mathrm{L}$ of this 'seed' solution was added to a 'growth' solution at room temperature. The latter was prepared by adding $0.10 \mathrm{ml} 0.10 \mathrm{M} \mathrm{L}$-ascorbic acid to $10.0 \mathrm{ml}$ aqueous solution containing $0.2 \mathrm{M} \mathrm{CTAB}, 0.5 \mathrm{mM}$ $\mathrm{HAuCl}_{4}$, and various concentrations of $\mathrm{AgNO}_{3}$ with stirring. The formation of gold nanorods proceeded over a period of several tens of minutes, and was associated with the development of a purple to blue color in the growth solution. The particles were purified by centrifugation and re-suspended. 


\section{RESULTS}

As is already well-known, the primary influence on the extinction efficiency of a nanorod is its aspect ratio, as shown in Figure 1. This can be readily controlled by variation of the concentration of $\mathrm{Ag}^{+}$added to the growth solution from 0 to $0.2 \mathrm{mM}$, which moves the position of the peak due to longitudinal plasmon resonance from $\sim 550 \mathrm{~nm}$ to $\sim 800 \mathrm{~nm}$, Figure 2. However, addition of more than $0.2 \mathrm{mM} \mathrm{Ag}^{+}$does not offer further improvement and decreases the rate of reaction considerably. A mixture of rods and spheres was present in all the samples, Figure 3, but a separation of the two particle forms can be achieved if desired by centrifugation. This is because the largest spheres settle fastest, the rods go down next, and any small nanospheres last of all.

Although aspect ratio is the dominant factor controlling the optical extinction of the particles, deviations in shape from prolate ellipsoidal, at a given length and aspect ratio, also have a quite significant effect on $Q_{\text {ext }}(\lambda)$, Figure 4 . We have explored this latter topic in greater depth elsewhere[2].

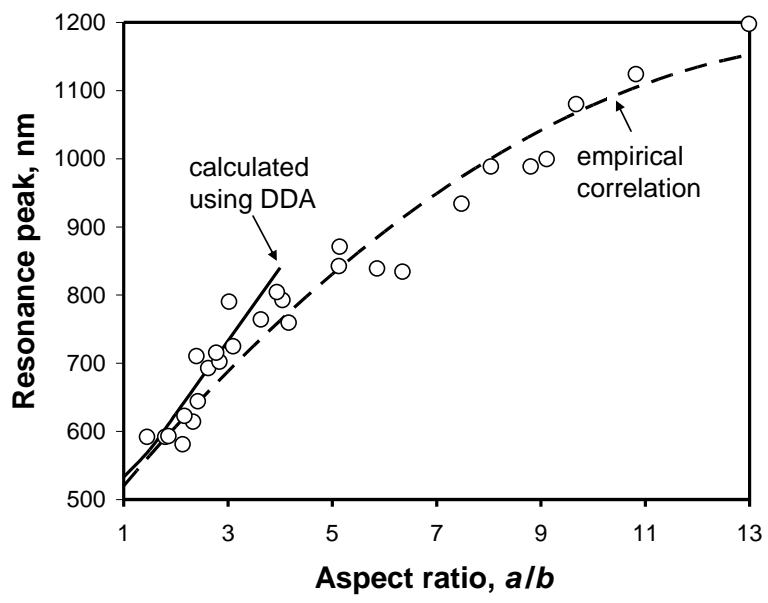

Figure 1. Effect of aspect ratio of a gold nanorod on the position of its peak optical extinction from Cortie[13], showing experimental data of Yu [14], a superimposed empirical correlation, and the trend from calculations using the DDA method.

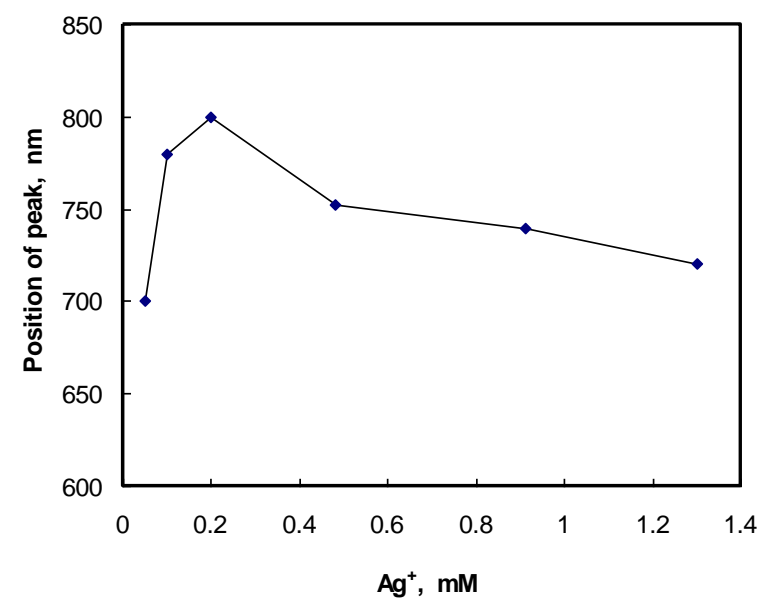

Figure 2. Effect of $\mathrm{Ag}^{+}$on the position of the maximum optical extinction.

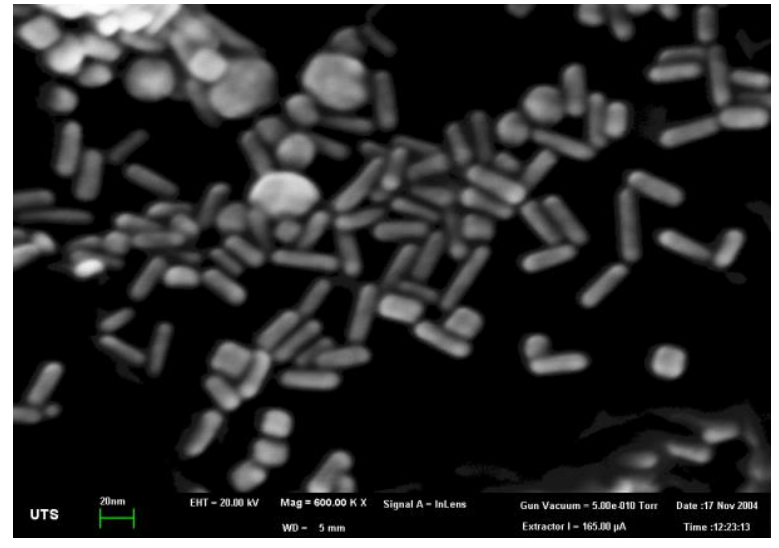

Figure 3. Particle distribution of rods and spheres produced with $0.2 \mathrm{mM}$ $\mathrm{Ag}^{+}$, shown here in the unpurified form.

The optical extinction of coatings of nanorods may also be modified by particle-to-particle interactions provided that the density of rods within the coating is sufficiently high to bring them to within a few tens of nanometres of one another. Endto-end configurations of rods cause a significant red-shifting and amplification of the longitudinal plasmon, a fact that has been recently verified experimentally [15]. However, when the rods are brought close together in other configurations the results are quantitatively and/or qualitatively different. Some aspects of this problem have been discussed elsewhere [16], and it was shown there, for example, that placing rods in a side-by-side configuration will blue-shift their peak absorption spectra. Here we extend the analysis of possible packing configurations by considering the case of rods in staggered and mutually perpendicular configurations, such as might also occur in closely-packed coatings of these particles. The various configurations are illustrated in Figure 5, and the results of DDA calculations are shown in Figures 6 and 7. In the present calculations the light is perpendicularly incident to the plane of Figure 5, with two orthogonal polarization directions.

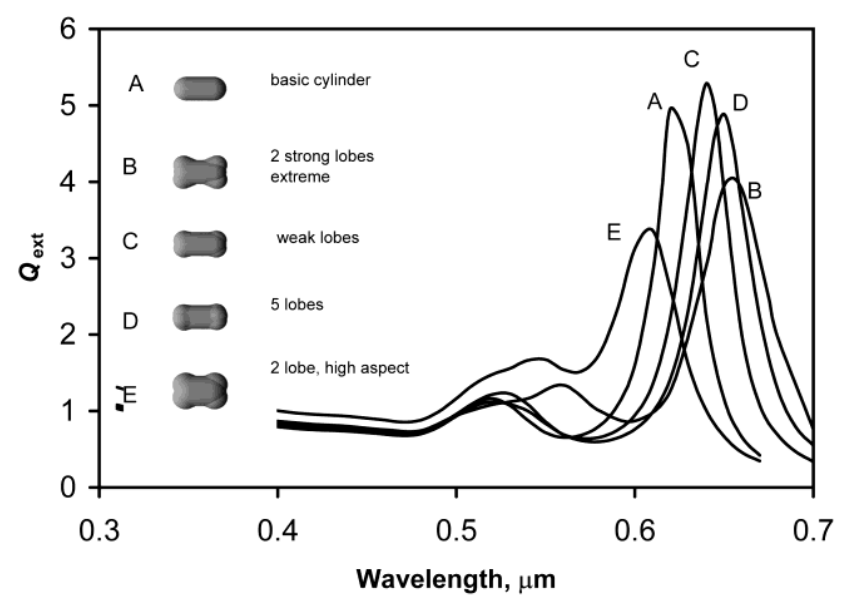

Figure 4. Effect of deviation in shape of a gold nanorod from hemispherecapped cylindrical on its optical extinction efficiencies 


\section{DISCUSSION}
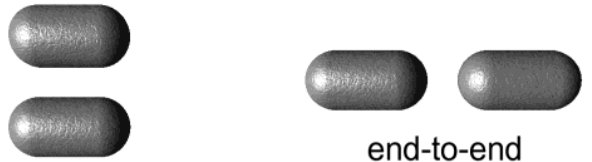

side-to-side

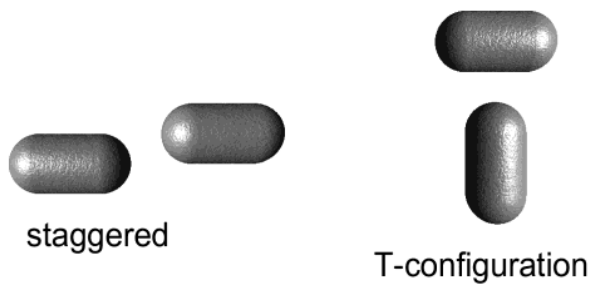

Figure 5. Schematic illustration of the various rod configurations discussed in the present work.

It is clear that the basic $625 \mathrm{~nm}$ resonance for a $2: 1$ hemisphere-capped cylinder can be modified to fall anywhere from 590 to $760 \mathrm{~nm}$ by dipole-dipole interactions between rods in close-packed coatings, while in Figure 2 we showed that changes in profile of the basic 2:1 shape can similarly cause the position of maximum extinction to vary between 600 and 650 nm. From Figure 1 it was evident that even small changes in aspect ratio had a strong effect on extinction. Taken together, these factors indicate that the extinction peaks of coatings comprised of a varied ensemble of rods, of for example a nominal 2:1 aspect ratio, will exhibit a broad extinction peak for light ranging in wavelengths between 600 and $700 \mathrm{~nm}$.

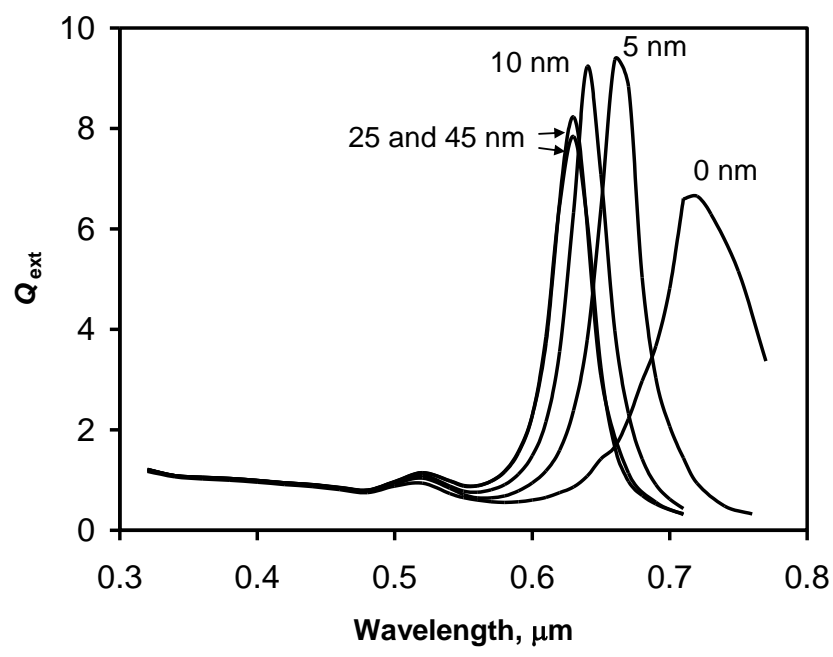

Figure 6. Red-shifting effect of rod-to-rod interactions in the staggered configuration.
A broad extinction peak may be desirable for solar window glazing, but is obviously not wanted in colour-change coatings, or in dichroic filters and polarizers. Use of gold nanorods in the latter would not only require rods of a very high degree of monodispersity but, as we have shown here, the packing of the rods in the coating must be such that a consistent dipole-dipole shift in plasmon resonance is achieved. The latter problem can of course be alleviated if the rods are dispersed in a threedimensional sense within a matrix. This is because, while the optical density of these coatings is strongly determined by the gold loading per unit of surface area of coating, the interparticle distances depend strongly on the thickness (and volume) of the coating. A two- or three-fold dilution of the rods with a transparent filler material may be all that is required to completely eliminate dipole-dipole effects.

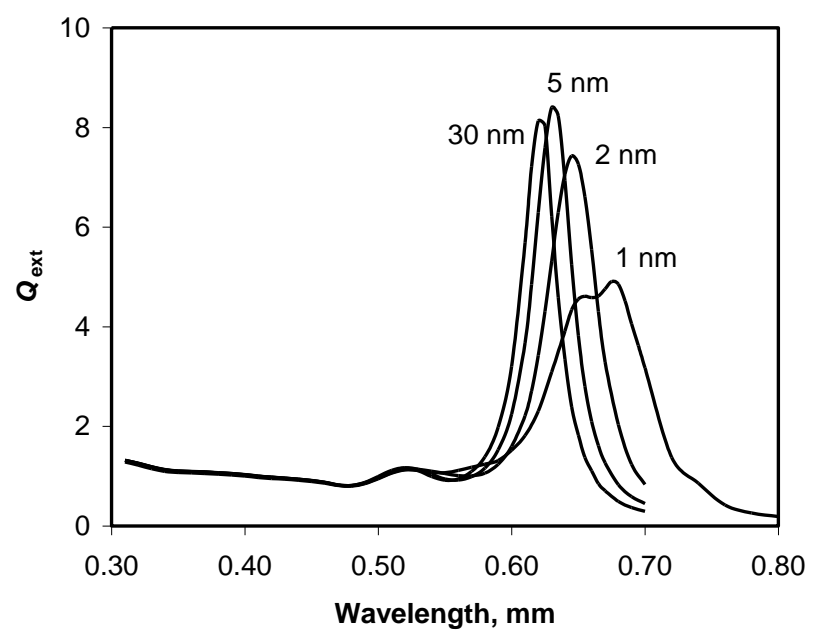

Figure 7. Red-shifting and attenuating effect of rod-to-rod interactions in the T-configuration

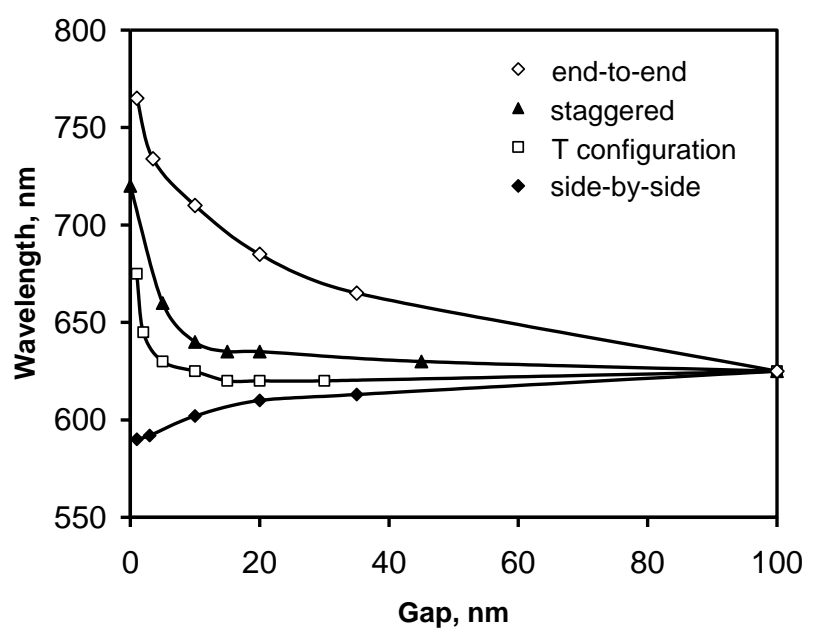

Figure 8. Trend in position of maximum optical extinction for the four different configurations defined in Figure 5. 


\section{CONCLUSIONS}

The optical extinction efficiency of gold nanorods shows two maxima, the first in the middle of the visible range, and the second at longer wavelengths. The first is caused by a transverse plasmon resonance, and the second by a longitudinal plasmon resonance. While it has long been known that the position of the latter is very sensitive to aspect ratio, we examine here how it is influenced by details of the rod morphology and by interactions with nearby rods. Three of the four rod-rod configurations examined here were associated with a red-shifting of the peak plasmon resonance. These factors will cause a significant broadening of the spectral selectivity of coatings comprised of nanorods.

\section{ACKNOWLEDGMENT}

The authors thank the Australian Research Council, the University of Technology Sydney, and AngloGold Ashanti Ltd for support. Computing resources were provided by the Australian Centre for Advanced Computing and Communication (ac3) in New South Wales and the national facility at the Australian Partnership for Advanced Computing (APAC).

\section{REFERENCES}

[1] J. Perez-Juste, I. Pastoriza-Santos, L. M. Liz-Marzan, and P. Mulvaney, "Gold nanorods: Synthesis, characterization and applications", Coordination Chemistry Reviews, vol. 249, pp.1870-1901, 2005.

[2] X. Xu and M. B. Cortie, "Shape change and color gamut in gold nanorods, dumbbells and dog-bones", Advanced Functional Materials, vol., pp.in press, 2006.

[3] M. B. Cortie, A. McDonagh, and P. Mulvaney, "Devices having a variable optical property and processes of making such devices", AU2005/201737 26th April 2005.

[4] C. J. Murphy, T. K. Sau, A. M. Gole, C. J. Orendorff, J. Gao, L. Gou, S. E. Hunyadi, and T. Li, "Anisotropic metal nanoparticles: synthesis, assembly, and optical applications", J. Phys. Chem. B, vol. 109, pp.13857-13870, 2005.

[5] J. K. N. Mbindyo, B. D. Reiss, B. R. Martin, C. D. Keating, M. J. Natan, and T. E. Mallouk, "DNA-directed assembly of gold nanowires on complementary surfaces", Advanced Materials, vol. 13, pp.249-254, 2001.

[6] N. Taub, O. Krichevski, and G. Markovich, "Growth of gold nanorods on surfaces", Journal of Phys. Chem. B, vol. 107, pp.11579-11582, 2003.

[7] Z. Wei, A. J. Mieszawska, and F. P. Zamborini, "Synthesis and manipulation of high aspect ratio gold nanorods grown directly on surfaces", Langmuir, vol. 20, pp.4322-4326, 2004.

[8] O.-H. Kwon, S. Lee, and D.-J. Janga, "Mesoscopic linear alignment and thermal-relaxation dynamics of aggregated gold nanorods", Eur. Phys. J. D, vol. 34, pp.243-246, 2005.

[9] K. E. Peceros, X. Xu, S. R. Bulcock, and M. B. Cortie, "Dipole-dipole plasmon interactions in gold-on-polystyrene composites", Journal of Physical Chemistry B, vol. 109, pp.21516 -21520, 2005.
[10] B. T. Draine and P. J. Flatau, "Discrete-dipole approximation for scattering calculations", J. Opt. Soc. Am. A, vol. 11, pp.1491-1499, 1994.

[11] B. T. Draine and P. J. Flatau, "User Guide to the Discrete Dipole Approximation Code DDSCAT 6.1", 2004, http://arxiv.org/abs/astroph/0409262, accessed January 2005.

[12] B. Nikoobakht and M. A. El-Sayed, "Preparation and growth mechanism of gold nanorods (NRs) using seed-mediated growth method", Chem. Mater., vol. 15, pp.1957-1962, 2003.

[13] M. Cortie, X. Xu, H. Zareie, H. Chowdhury, and G. Smith, in Smart Materials, Nano-, and Micro-Smart Systems II, Vol. 5649 (S. F. AlSarawi, ed.), SPIE, Sydney, Australia, 2005, p. 565-573.

[14] Y.-Y. Yu, S.-S. Chang, C.-L. Lee, and C. R. C. Wang, "Gold nanorods: Electrochemical synthesis and optical properties", Journal of Physical Chemistry B, vol. 101, pp.6661-6664, 1997.

[15] K. G. Thomas, S. Barazzouk, B. I. Ipe, S. T. S. Joseph, and P. V. Kamat, "Uniaxial plasmon coupling through longitudinal self-assembly of gold nanorods", Journal of Physical Chemistry B, vol. 108, pp.13066-13068, 2004.

[16] M. B. Cortie, X. Xu, and M. J. Ford, "Effect of composition and packing configuration on the dichroic optical properties of coinage metal nanorods", Physical Chemistry : Chemical Physics, vol. 8, pp.35203527,2006 